\title{
Optimization of the power consumption mode of pumping stations of "Suv Okova" by reactive power
}

\author{
Shavkat Muzafarov, Abdusaid Isakov", Ruzimurat Choriev, Zukhra Ismailova and Durdona \\ Mustafoyeva \\ Tashkent Institute of Irrigation and Agricultural Mechanization Engineers, Tashkent, Uzbekistan
}

\begin{abstract}
The optimal modes of the existing compensating devices under operating conditions were determined. Minimum power and energy losses were taken as optimality criteria. The unconstrained optimization problem was considered, i.e., finding the absolute minimum. When solving the problem to find the optimal mode of operation of the "SUV OKOVA" network, a relative minimum was found since the system of constraints has a nonlinear form. The problem of conditional optimization in terms of reactive power is solved, for which the gradient method of quadratic programming is used.
\end{abstract}

\section{Introduction}

In recent years, the rapid development of the power industry has posed new challenges for optimizing reactive power in an active distribution network. Traditional stochastic optimization and robust optimization techniques can lead to overly conservative or overly risky decisions when dealing with renewable energy uncertainties. In [1,2], a distributionally robust reactive power optimization model for an active distribution network was proposed; it considers network losses as an aim of optimization and considers reactive power support and switch reconfiguration. A data-driven two-step reactive power optimization model with stable distribution is developed. The model of hybrid optimization of several renewable energy sources used to evaluate the primary data, the development of a load profile, and the determination of the optimal option for the system with the lowest cost was discussed in $[3,4]$. Methods for determining the size of distributed generation units (DG) and the assessment of optimal locations in electrical distribution networks were proposed in [5-7]. To connect DG, some basic optimization principles were introduced, and examples of optimization of the size and location of DG units were presented.

A formalized mathematical description of the optimization problem was considered in [8-10]. Constraints in the mathematical description represent various technical, economic, environmental conditions taken into account when solving the optimization problem [9-12]. Mathematical programming is, as a rule, a repetitive computational procedure leading to the desired optimal solution [9-12]; for finding an extremum point, it is impossible or very difficult to use analytical methods of solution, in that case, a nonlinear programming

* Corresponding author: abdusaidus72@mail.ru 
method is used to solve optimization problems. As a rule, when solving problems by nonlinear programming methods, numerical methods are used [11-19].

\section{Methods and Materials}

\subsection{Reactive power balance equation}

The total power of the compensating devices (CD) required in the electric power system (EPS) is determined from the reactive power balance equation. This power must be placed in the nodes of the electrical network with minimal costs.

$$
\Sigma Q_{G}=\Sigma Q_{H}+\Sigma Q_{C H}+\Delta Q_{\Sigma}=\Sigma Q_{P}
$$

Where $\Sigma Q_{G}$ is the total reactive power generated in the EPS, including the reactive power transmitted from the neighboring EPS; $\sum Q_{H}$ is the total reactive power of EPS consumers, including reactive power, transmitted to the neighboring EPS; $\sum Q_{C H}$ is the total reactive power of auxiliary needs of power plants; $\Delta Q_{\Sigma}$ are the total losses of reactive power; $\sum Q_{\Pi}$ is the total consumption of reactive power in the EPS.

Let us consider the simplest scheme of the existing network (Fig.1). The load with power $S=P+j Q$ receives power from the source with voltage $U$ through the network resistance $R$ [18]. A compensating device with the power $Q_{k}$ is installed on the load buses.

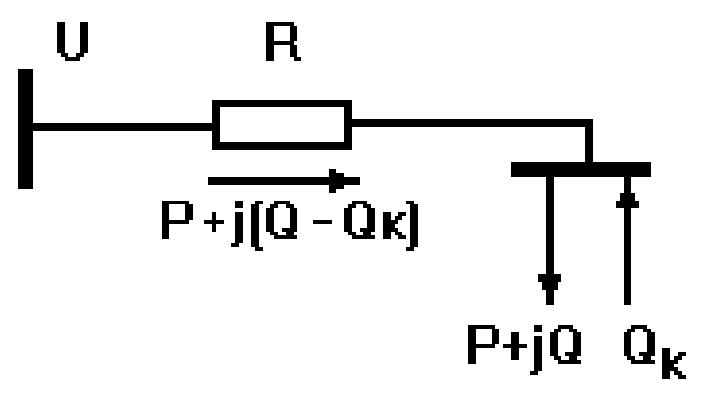

Fig. 1. The simplest circuit of reactive power compensation

Losses of active power in the line in the absence of a compensating device ( $\left.Q_{k}=0\right)$ for the consumer are

$$
\Delta P=\frac{\left(P^{2}+Q^{2}\right) R}{U^{2}}
$$

When a compensating device $\left(Q_{k} \neq 0\right)$ is installed for the consumer, these losses decrease to the following value:

$$
\Delta P=\frac{\left(P^{2}+\left(Q-Q_{k}\right)^{2}\right) R}{U^{2}}
$$


Thus, the compensation of reactive power allows us to reduce the losses of active power in the power supply circuit and, consequently, to improve the technical and economic indicators of this circuit.

Impact of CD on network costs. Let us estimate the impact of CD on network costs. The expression for the total costs of transferring power to the load during the installation of $\mathrm{CB}$ has the form:

$$
3=3_{K}+c_{o} \Delta P=3_{K} Q_{K}+c_{o} \frac{\left(P^{2}+\left(Q-Q_{K}\right)^{2}\right) R}{U^{2}},
$$

where $3_{K}$ is the cost for $\mathrm{CD} ; c_{o} \Delta P$ are the costs of covering active power losses in the network; $c_{o}$ is the cost of a unit of lost active power; $3_{\kappa}$ are the unit costs for CD.

To determine the minimum of function 3 , we equate its derivative of the variable $Q_{K}$ to zero:

$$
\frac{d 3}{d Q_{K}}=3_{\kappa}-\frac{2\left(Q-Q_{K}\right) R c_{o}}{U^{2}}=0
$$

The economically feasible reactive power is determined from (4); its transfer from the source to the consumer meets the minimum costs 3 .

$$
Q_{\ni}=Q-Q_{K}=\frac{{ }_{3} U^{2}}{2 R c_{o}} \text {. }
$$

The value of $Q_{\ni}$ does not depend on the active power $P$ but depends only on the ratio of the cost indicators $3_{K}$ and $c_{o}$ and the parameters of the network $U$ and $R$ through which the power is transmitted.

Location of CD in power systems. The issue of the location of compensating devices in the electrical network of a real EPS is a complex optimization problem. The difficulty lies in the fact that electric power systems are large systems of interconnected subsystems. It is impossible to consider each individual subsystem since the properties of large systems are determined by the nature of the interconnections of individual subsystems.

In the analysis of large systems, a systematic approach is used [18,19], according to which the analysis of a large system is performed by dividing it into subsystems that are not directly related to each other but affect each other through a higher-level system. 


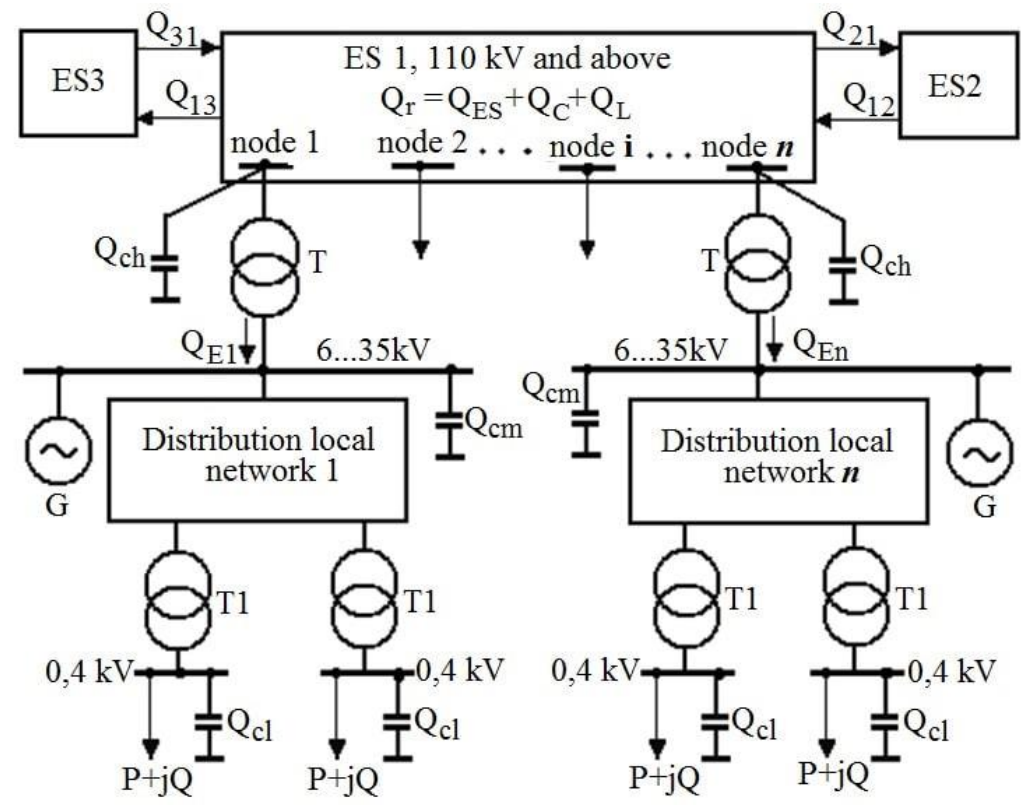

Fig. 2. The location of CD devices in the electrical network

With regard to the issue under consideration, the electrical network is represented by different levels, as shown in Fig.2. The upper level is an electrical network with a voltage of $110 \mathrm{kV}$ and higher. This complex electrical network, represented by a complete equivalent circuit, is shown in Fig.2, conventionally, as ES1. Reactive powers generated by power plants generators $Q_{\ni}$, compensating devices $Q_{K}$, and power lines $Q_{C}$, and reactive powers flowing through connections with neighboring ES2 and $\operatorname{ES} 3\left(Q_{12}, Q_{21}, Q_{13}, Q_{31}\right)$ provide available reactive power $Q_{p l}$ in ES1.

The second level is a set of $n$ open local distribution networks with a voltage of $35 \mathrm{kV}$ and lower, connected to $n$ nodes of the upper-level electrical network through transformers $T$. These local distribution networks are not directly connected to each other but affect each other through the upper-level network. Synchronous generators, compensators, and motors in each distribution network are represented by one equivalent synchronous machine $G$. Low-voltage consumers $P+j Q$ are supplied from local electrical networks through distribution transformers $\mathrm{T} 1$.

Compensating devices can be installed on the buses of higher $\left(j Q_{\kappa \varepsilon}\right)$ and lower $\left(j Q_{\kappa c}\right)$ voltage of transformers $\mathrm{T}$, and on the buses of $0.4 \mathrm{kV}$ of distribution transformers $\mathrm{T} 1$ and in the $0.4 \mathrm{kV}$ network itself $\left(j Q_{\kappa н}\right)$. The value of the CD power is to be determined.

In general, the problem of optimizing the $\mathrm{CD}$ location is formulated as follows: to determine the reactive powers in the nodes $6 \ldots 35 \mathrm{kV}$ of the synchronous machines $\mathrm{G}$, the power of CD in the networks of all voltages $Q_{\kappa B}, Q_{\kappa c}, Q_{\kappa H}$, and the values of the reactive powers $Q_{\ni i}(\mathrm{i}=1,2, \ldots n)$, transmitted in the consumers' networks, which ensure the minimum total costs.

Calculations of reactive power compensation for networks of all types are carried out both when designing the development of electrical networks and their operation conditions. In the process of designing, the $\mathrm{CD}$ power is determined, and the problem of their distribution in the electrical network is solved. Under operating conditions, the optimal modes of the existing CD are determined within 24 hours. Optimality criteria, in this case, 
are the minimum power and energy losses and the compliance of voltage deviations with permissible values.

When designing a power supply scheme, the monetary costs for this scheme are minimized as a rule. Reducing power losses due to the installation of CD reduces the costs of the circuit, for the following reasons:

- every lost $\mathrm{kW}$ of power was generated at power plants and, therefore, money was spent on it;

- generation of lost reactive power at power plants is much more expensive (by 3 times!) than consumption.

However, compensating devices are also expensive.

\section{Results and Discussion}

Determination of the optimal power of CD. In this regard, the problem arises of determining the optimal power of compensating devices that meets the minimum total costs. This problem belongs to the problem of unconstrained optimization and can be solved, for example, by gradient methods.

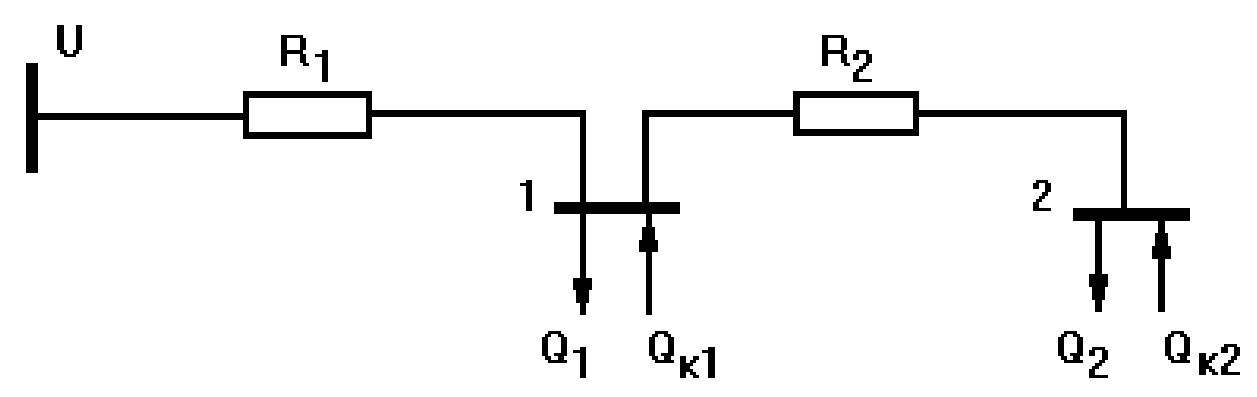

Fig. 3. Power supply scheme

Consider such a problem for the main power supply circuit (Fig.3). It is necessary to determine the power of the compensating devices $\mathrm{Q}_{\mathrm{K} 1}$ and $\mathrm{Q}_{\mathrm{K} 2}$ in nodes 1 and 2 based on the condition of the minimum total costs for the installation of these devices and the coverage of active power losses in the circuit.

Initial data:

circuit voltage $U$;

resistance of lines $R_{1}$ and $R_{2}$;

reactive loads of nodes 1 and $2 Q_{1}$ and $Q_{2}$;

unit costs for the installation of compensating devices $z_{o}$;

unit costs to cover active power losses $c_{o}$.

The target function, which is the total costs of installing compensating devices and covering active power losses in the circuit, has the following form

$$
Z=z_{0}\left(Q_{k 1}+Q_{k 2}\right)+a_{1}\left(Q_{1}+Q_{2}-Q_{k 1}-Q_{k 2}\right)^{2}+a_{2}\left(Q_{2}-Q_{k 2}\right)^{2} \rightarrow \min
$$

where $\mathrm{a}_{1}=\mathrm{R}_{1} \cdot \mathrm{c}_{\mathrm{o}} \cdot 10^{-3} / \mathrm{U}^{2}=0.0006 ; \mathrm{a}_{2}=\mathrm{R}_{2} \cdot \mathrm{c}_{\mathrm{o}} \cdot 10^{-3} / \mathrm{U}^{2}=0.0004$.

The introduction of a numerical coefficient $10^{-3}$ is necessary to reduce all components of the target function to one dimension (c.u.).

To solve the problem, we choose the coordinate descent method. Let us define the partial derivatives of the target function $\mathrm{Z}$ concerning the variables $Q_{l}$ and $Q_{2}$ : 


$$
\begin{gathered}
\frac{\partial Z}{\partial Q_{k 1}}=z_{0}-2 a_{1}\left(Q_{1}+Q_{2}-Q_{k 1}-Q_{k 2}\right), \\
\frac{\partial Z}{\partial Q_{k 2}}=z_{0}-2 a_{1}\left(Q_{1}+Q_{2}-Q_{k 1}-Q_{k 2}\right)-2 a_{2}\left(Q_{2}-Q_{k 2}\right) .
\end{gathered}
$$

The initial approximation is taken as:

$$
\begin{aligned}
& Q_{k 1}{ }^{0}=0, \\
& Q_{k 2}{ }^{0}=0 .
\end{aligned}
$$

For these values, we calculate the target function and its partial derivatives.

We assume that the objective function $\mathrm{Z}$ decreases more strongly in the direction of the variable $Q_{k 2}$ than in the direction of the variable $Q_{k 1}$, i.e.

$$
\left|\frac{\partial Z}{\partial Q_{k 2}}\right|>\left|\frac{\partial Z}{\partial Q_{k 1}}\right| .
$$

The descent starts in the direction of the variable $\mathrm{Q}_{\mathrm{k} 2}$.

Let us take the step size $\lambda=400 \mathrm{kVAr}$. The first approximation (first step) is $Q_{k l}{ }^{1}=0$, $Q_{k 2}{ }^{l}=400 \mathrm{kVAr}$. We calculate the value of the target function $Z^{l}$.

The second step is $Q_{k 1}{ }^{2}=0, Q_{k 2}{ }^{2}=400 \mathrm{kVAr}$. We calculate the value of the target function $Z^{2}$. The descent along the $Q_{k 2}$ coordinate should be continued until $Z^{n}<Z^{n-1}$. As soon as $Z^{n}$ becomes greater than the previous value of $Z^{\mathrm{n}-1}$, the descent along the $Q_{k 2}$ coordinate should be stopped and returned to the values of the variables $Q_{k 1}^{n-1}$ and $Q_{k 2}{ }^{n-1}$, obtained at the $n-1$ step.

Let us take a new step towards another variable $Q_{k l}$. The new value of the target function $\mathrm{Z}$ is found. The descent along this variable continues in the same way as in the direction of $Q_{k 2}$-until $Z^{m}<Z^{m-1}$.

The point with the obtained coordinates $Q_{k 1}{ }^{m-1}, Q_{k 2}{ }^{n-1}$ is in the vicinity of the minimum of the target function $\mathrm{Z}$. With the set step length $\lambda=400 \mathrm{kVAr}$, a more exact solution cannot be obtained. To obtain a more exact solution, it is necessary to decrease the step and continue the descent. It is absolutely certain that the less the step, the more exact the result. We cannot achieve this accuracy through hand calculation. To solve this problem, it is advisable to use software designed to solve the problem of nonlinear programming with nonlinear constraints. One of these programming languages is $\mathrm{C}++$.

This was the consideration of the problem of unconstrained optimization, i.e., finding the absolute minimum. When solving the problem, to find the optimal operating mode for the "SUV OKOVA" network, it is necessary to find a relative minimum since the system of constraints has a nonlinear form. Thus, the problem of conditional optimization in terms of reactive power is posed, for which we apply the previously selected gradient method of quadratic programming. 


\section{Conclusions}

1. The total power of the compensating devices required in the electric power system (EPS) is determined using the reactive power balance equation.

2. Under operating conditions, the optimal modes of the existing $\mathrm{CD}$ are determined within 24 hours. Optimality criteria, in this case, are the minimum power and energy losses and compliance of voltage deviations with permissible values.

3. The target function is the total cost of installing compensating devices and coverage of active power losses in the circuit.

4. The problem of unconstrained optimization, i.e. finding the absolute minimum is considered. When solving the problem, to find the optimal operating mode for the "SUV OKOVA" network, it is required to find a relative minimum since the system of constraints has a nonlinear form. Thus, we are faced with the problem of conditional optimization in reactive power, for which we apply the gradient method of quadratic programming.

\section{References}

1. Hebin Ruan, Hong Jun Gao. Jun Yong Liu, You Bo Liu A distributionally robust reactive power optimization model for active distribution network considering reactive power support of $\mathrm{dg}$ and switch reconfiguration, pp. 6358-6365, (2019)

2. Isakov A., Abdullaev Z. Study on increase of operation efficiency of electrical energy and electrical equipment. 2020 IOP Conf. Ser.: Earth and Environmental Science 614(1) 012047.

3. Barry Haves. Chapter 9-distribution generation optimization and energy management. distributed generation systems. design, operation and grid integration 2017, pages 415-451.

4. Isakov A., Rakhmatov A. Control over parameters of ionized air. 2020 IOP Conf. Ser.: Mater. Sci. Eng. 883(1) 012118

5. Gebrehiwot Kiflom, Alam Md., Mondal H., Ringler C., Getaneh G., Optimization and cost-benefit assessment of hybrid power systems for off-grid rural electrification in Ethiopia. Journal Energy. Volume 177. 15 june 2019. Pp. 234-246.

6. Muzafarov S., Balitskiy V., Toqaev B., Batirova L., Isakov A. The research results of cleaning air stream process from aerosol particles in electric fields of corona discharge stream form. 2020 IOP Conf. Ser.: Earth and Environmental Science 614(1) 012008.

7. Mirzabaev A., Isakov A., Mirzabekov S., Makhkamov T., Kodirov D. Problems of integration of the photovoltaic power stations with the grid systems. 2020 IOP Conf. Ser.: Earth and Environmental Science 614(1) 012016.

8. Kostin V. Optimization tasks of the electric power industry: teaching aid. Petersburg: sztu, 2003. -120p.

9. Chernorutskiy I. Optimization methods in control theory. Petersburg peter, 2004 226p.

10. Isakov A., Rakhmatov A., Ismailova Z. Study the effect of the design of discharge electrodes on the characteristics of the corona discharge. 2020 IOP Conf. Ser.: Earth and Environmental Science 614(1) 012011.

11. Kostin V., Raspopov E., Rodchenko E. Electricity transmission and distribution: teaching aid. Petersburg: sztu, $2003-147$ p.

12. Isakov A., Mirzabaev A., Sitdikov O., Makhkamova M., Kodirov D. Innovative methods of developing solar power systems for remote and agricultural facilities in Uzbekistan. 2020 IOP Conf. Ser.: Earth and Environmental Science 614(1) 012014.

13. Barzam A. System automation. - M.: Energoatomizdat, 1989. -446p. 
14. Radjabov A., Ibragimov M., Eshpulatov N.M., Matchonov, O. Improving the energy performance of ozone generators used in agricultural ecology. Journal of Physics: Conference Series. Volume 1399, Issue 5, 5 December 2019, 055060.

15. Barkan Ya., Orekhov L. Automation of power systems: a textbook for university students. -M.: Higher School, 1981. -271p.

16. Ibragimov M., Eshpulatov N., Matchanov O. Electrical technology of moisture content reduction of industrial-grade cotton seeds, IOP Conference Series: Materials Science and Engineering. 883(1), (2020)

17. Ibragimov M., Eshpulatov N., Matchanov O. Substantiation of the optimal parameters by processing with electric contact methods to decrease the moisture content of technical seeds. IOP Conf. Series: Earth and Environmental Science. 614 (2020) 